\title{
Boycotts, bombs, or bargains? Eine Analyse von Strategien im Umgang mit Nordkoreas Nuklearwaffenprogramm
}

\author{
Gerhard Mangott • Martin Senn
}

Online publiziert: 30. Januar 2017

(C) Der/die Autor(en) 2017. Dieser Artikel ist eine Open-Access-Publikation.

Zusammenfassung Der Artikel diskutiert Strategien im Umgang mit Nordkoreas Nuklearwaffenprogramm. Das zentrale Argument lautet, dass schrittweises engagement, das positive Anreize an die Umsetzung mehrerer Phasen von Rüstungskontrolle bindet, die aussichtsreichste Strategie hinsichtlich einer Verhaltensänderung Nordkoreas darstellt. Strategien der Resignation, der Sanktion und der militärischen Eskalation werden als nicht zielführend oder zu risikoreich abgehandelt. Auf absehbare Zeit kann demnach lediglich das Ausmaß des nordkoreanischen Nuklearwaffenprogrammes begrenzt werden, während dessen Existenz nicht mehr Verhandlungsgegenstand sein kann.

Schlüsselwörter Nordkorea · Nuklearwaffen · Strategien · Sanktionen · Engagement

\section{Boycotts, Bombs or Bargains? An Analysis of Strategies in Dealing with North Korea's Nuclear Weapons Program}

\begin{abstract}
The article discusses strategies in dealing with the nuclear-weapons program of North Korea. It argues that a strategy of engagement, which links incentives to the implementation of various measures of arms control, is the most promising strategy to alter the behaviour of the DPRK. Strategies of resignation, sanctions and mililtary escalation are considered not feasible or too risky. For the forseeable future, only the extent of the DPRK's nuclear program can be limited, its very existence can no longer be negotiated.
\end{abstract}

Prof. Dr. G. Mangott $(\varangle) \cdot$ Assoz. Prof. Dr. M. Senn $(\bowtie)$

Institut für Politikwissenschaft, Universität Innsbruck, Universitätsstraße 15, 6020 Innsbruck, Österreich

E-Mail: gerhard.mangott@uibk.ac.at; martin.senn@uibk.ac.at 
Keywords North Korea $\cdot$ Nuclear weapons $\cdot$ Strategies $\cdot$ Sanctions $\cdot$ Engagement

\section{Einleitung}

Der vierte und fünfte Test eines nuklearen Sprengsatzes durch Nordkorea im Januar und September 2016, mehrfache Tests von Trägerraketen verschiedener Reichweiten und die anhaltend eskalierende Rhetorik des Regimes verdeutlichen, dass der ostasiatische Paria-Staat sein Nuklearwaffenprogramm entschlossen vorantreibt auch gegen erheblichen internationalen Widerstand und angesichts des Risikos, dass dieses Verhalten schließlich auch China als letzte Schutzmacht vergrämen könnte. Entgegen den offiziellen Verlautbarungen nordkoreanischer Medien ist es zwar wenig wahrscheinlich, dass Nordkorea an hochentwickelten Fusionswaffen arbeitet, jedoch scheint es Technologien zu entwickeln, die eine Verkleinerung nuklearer Sprengsätze und damit deren Transport mit ballistischen Trägermitteln ermöglichen ${ }^{1}$.

Angesichts der Risiken einer regionalen Destabilisierung durch Nordkoreas aggressives Gebaren und der Weiterverbreitung nordkoreanischer Technologien ist die zentrale Frage, ob und wie sich die weitere Entwicklung des Nuklearwaffen- und Raketenprogramms eindämmen und, bestenfalls, umkehren lässt. Als Antwort auf diese Frage nach effektiven Strategien im Umgang mit Nordkoreas Verhalten argumentiert der vorliegende Beitrag, dass schrittweises engagement, das positive Anreize an die Umsetzung mehrerer Phasen von Rüstungskontrolle bindet, die aussichtsreichste Strategie hinsichtlich einer Verhaltensänderung darstellt. Eine solche Strategie müsste jedoch mit der Prämisse der gegenwärtigen Sanktionierungs-Strategie brechen, die vom Ziel einer unmittelbaren und umfassenden nuklearen Abrüstung auf der koreanischen Halbinsel ausgeht. Die Prämisse einer neuen Strategie des engagement müsste vielmehr sein, dass auf absehbare Zeit lediglich das Ausmaß des nordkoreanischen Nuklearwaffen- und Raketenprogrammes begrenzt werden kann. Gleichwohl müsste Abrüstung als Fernziel erhalten bleiben, um eine de-facto-Anerkennung Nordkoreas als Nuklearwaffenstaat zu verhindern.

Der Beitrag entwickelt dieses Argument in vier Schritten. In einem ersten Schritt setzt er sich mit der Motivlage Nordkoreas zur Aneignung von Nuklearwaffen und ballistischen Trägermitteln auseinander. In einem zweiten Schritt führt er ein Spektrum von Strategien zur Änderung des nordkoreanischen Verhaltens ein, das von Resignation bis zu militärischer Intervention reicht und beleuchtet die jeweiligen Motive und Möglichkeiten der Großmächte, die sich seit geraumer Zeit vorrangig um eine Denuklearisierung Nordkoreas bemühen. Der Beitrag geht hierbei zunächst auf die Strategien der Resignation, Intervention und Sanktion ein und analysiert,

\footnotetext{
${ }^{1}$ Die Sprengkraft der beiden jüngsten Tests lag im Bereich von 10-20 Kiltonnen (10.000-20.000 Tonnen TNT), während die Sprengkraft einer Fusionswaffe (oder Wasserstoffbombe) weitaus größer ist. So hatte der erste Test einer Fusionswaffe durch die Vereinigten Staaten von Amerika eine Sprengkraft von 10 Megatonnen (10 Mio. Tonnen TNT) und der W76 Gefechtskopf der USA hat eine Sprengkraft von ungefähr 100 Kilotonnen. Da die Konstruktion von Fusionswaffen technolgisch anspruchsvoll ist, erscheint es plausibler, dass Nordkorea an einem sogenannten boosted-fission Design arbeitet. Dieses Design nutzt die Fusion von Wasserstoff Isotopen für eine Verstärkung einer nuklearen Kettenreaktion und damit einer Erhöhung der Sprengkraft.
} 
warum diese nicht zu einer nachhaltigen Verhaltensänderung beitragen können. In einem dritten Schritt konzentriert sich der Beitrag schließlich auf Engagement-Strategien, wobei er zwischen engagement als grand bargain und schrittweisem engagement unterscheidet, das Anreize mit Rüstungskontrolle verbindet. Der Beitrag schließt, viertens, mit dem Befund ab, dass ein grand bargain wenig aussichtsreich ist, schrittweises engagement jedoch einen Hebel zur Änderung des nordkoreanischen Verhaltens darstellt, auch wenn dieses ebenfalls mit einer Reihe von Herausforderungen einhergeht.

\section{Die Motive Nordkoreas - Abschreckung, Erpressung, Herrschaftssicherung}

Die Forschung zur Frage, warum sich Staaten Nuklearwaffen aneignen, identifizierte über lange Zeit hinweg das Streben nach Sicherheit in einem anarchischen Umfeld als zentrales Motiv für nukleare Weiterverbreitung (Paul 2000; Waltz 1981). Erst in jüngerer Zeit konzentrieren sich WissenschaftlerInnen vermehrt auf weitere, innerstaatliche Motive wie etwa das Streben nach Regimesicherheit (Solingen 2007) oder nationale Identitätsvorstellungen (Hymans 2006). Die Entscheidung für oder gegen Nuklearwaffen ist demnach multikausal (Sagan 1996/1997, S. 85) sie ergibt sich aus einem komplexen Zusammenspiel außen- und innenpolitischer Motive $^{2}$. Dies gilt auch für den Fall Nordkoreas, dessen Nuklearwaffenprogramm der Abschreckung externer Bedrohungen, der Erpressung von Zugeständnissen und Hilfeleistungen externer Akteure sowie der Konsolidierung des Regimes dient.

Das Kim-Regime sieht das Land in einem permanenten Belagerungszustand und existenziellen, anti-imperialistischen Kampf, der zunächst gegen den japanischen Imperialismus geführt wurde und seit dem Korea-Krieg gegen den US-amerikanischen Imperialismus gerichtet ist. Die Vereinigten Staaten werden als entschlossener Feind perzipiert, der bereits während des Korea-Krieges den Einsatz von Nuklearwaffen erwog, über Jahrzehnte taktische Nuklearwaffen auf der koreanischen Halbinsel stationierte und gemeinsam mit Südkorea jährliche Großmanöver durchführt (Bleiker 2003, S. 725-726)3. Nachhaltig verstärkt wurde diese Wahrnehmung einer existenziellen Bedrohung durch die Rogue-State-Rhetorik, derer sich amerikanische EntscheidungsträgerInnen seit den frühen 1990er-Jahren in zunehmenden Maße bedienten sowie durch die Regimewechselpolitik der George W. Bush Administration, die Nordkorea (gemeinsam mit Iran und Irak) zudem als Teil einer axis of evil deklarierte. Es ist daher auch wenig überraschend, wenn offizielle Kommentare nach dem jüngsten Nuklearwaffentest betonen, dass die Führung in Pjöngjang vom Schicksal Saddam Husseins und Muammar Al Gaddafis gelernt habe: Diese hätten unter Druck

\footnotetext{
2 Siehe Senn (2013) für eine kritische Bestandsaufnahme der Literatur zur Anreizstruktur (demand-side) nuklearer Weiterverbreitung.

3 Siehe ebenfalls den Artikel DPRK's H-bomb test logical chain of reaction, der am 14. Januar in der Pyongyang Times erschien (Pyongyang Times 2016). Wie Sigal (2011, S. 24) in diesem Zusammenhang anmerkt: „No country has been the target of more US nuclear threats than North Korea - at least seven since 1945.“
} 
von außen ihre Nuklearwaffenprogramme aufgegeben und wären damit der Regimewechsel-Politik der Vereinigten Staaten und ihrer Verbündeten schutzlos ausgeliefert gewesen (Korean Central News Agency 2016b).

Das Kim-Regime sieht ein Arsenal an Nuklearwaffen und ballistischen Trägermitteln demnach als den ultimativen und langfristigen Garanten souveräner Existenz angesichts eines andauernden Konfliktes mit übermächtigen imperialistischen Kräften. Diese Rolle von Nuklearwaffen ist umso wichtiger als die konventionellen Streitkräfte Nordkoreas mit gravierenden Herausforderungen konfrontiert zu sein scheinen. Zwar weisen die Streitkräfte eine massive personelle Stärke von mehr als einer Millionen aktiver SoldatInnen auf (International Institute for Strategic Studies 2016, S. 264), jedoch haben sie gleichzeitig mit einer Überalterung des Geräts sowie mit Problemen in der Versorgung mit Nahrung und Treibstoffen zu kämpfen (Cordesman und Lin 2015, S. 79; IISS 2016, S. 264; Chanlett-Avery et al. 2016, S. 17-18). Diese Herausforderungen lassen die Bestandsfähigkeit der nordkoreanischen Streitkräfte gegen die Streitkräfte Südkoreas (vor allem in einem Verbund mit den Vereinigten Staaten) zweifelhaft erscheinen.

In der Abwehr externer Bedrohungen spielt neben der Weiterentwicklung der materiellen Fähigkeiten im Bereich von Nuklearwaffen und ballistischen Trägermitteln auch die gezielte Eskalation durch Waffentests, Grenzscharmützel und martialische Rhetorik eine maßgebliche Rolle. Das Kim-Regime hat über Jahrzehnte das Bild eines reizbaren, unberechenbaren und zu allem entschlossenen Akteurs gepflegt. So verlautbarte es etwa wiederholte Male, dass es Seoul in ein Flammenmeer verwandeln wolle und veröffentlichte jüngst ein Video, das einen nuklearen Angriff auf Washington, D.C. darstellt (Stimme Koreas 2016) ${ }^{4}$. Dieses Bild eines irrationalen Akteurs fällt vor allem in westlichen Staaten auf fruchtbaren Boden, in denen das Schreckgespenst eines Irren mit der Bombe von jeher Teil des Denkens über Nuklearwaffen ist. Diese Furcht vor dem Gegenteil des (vermeintlich) rationalen Besitzers von Nuklearwaffen, wie er in den etablierten Nuklearwaffenstaaten gesehen wird, wird nicht nur durch die Erwartung genährt, dass EntscheidungsträgerInnen in neuen Nuklearwaffenstaaten individuelle Pathologien aufweisen könnten, sondern auch durch die Annahme, dass Staaten in nicht-westlichen Kulturkreisen weniger verantwortungsvoll im Umgang mit Nuklearwaffen seien (Gusterson 1999). Eben diesen „nuklearen Orientalismus“ (Gusterson 1999, S. 113-116) bedient die nordkoreanische Führung mit ihrem eskalierenden Verhalten.

Nordkorea versucht dadurch jedoch nicht nur die Glaubwürdigkeit seiner Abschreckungsfähigkeit gegenüber den militärisch um ein Vielfaches stärkeren Vereinigten Staaten zu erhöhen, sondern auch Zugeständnisse anderer Staaten zu erzwingen. So folgte auf den letzten Nuklearwaffentest im Januar 2016 neuerlich die Drohung, dass Nordkorea die Tests so lange fortführen werde, bis die Vereinigten Staaten zur Unterzeichnung eines Friedensvertrages bereit seien. Die Angst vor einer erratischen Führung in Pjöngjang vor weiterer regionaler Eskalation sowie vor der Proliferation nordkoreanischer Technologie hat Großmächte wie Nachbarstaaten in

\footnotetext{
4 Das Video enthält einen Untertitel, in dem Nordkorea ankündigt: „If the American imperialists provoke us a bit, we will not hesitate to slap them with a pre-emptive nuclear strike“. Siehe hierzu Sang-Hun (2016).
} 
der Vergangenheit immer wieder an den Verhandlungstisch geführt und Nordkorea damit Nahrungsmittel, Energie und humanitäre Hilfe eingebracht ${ }^{5}$.

Zwar ist die Wahrung der souveränen Existenz durch Abschreckung und Erpressung ein zentrales Motiv für Nordkoreas Besitz von Nuklearwaffen, jedoch hat dieser auch wichtige innenpolitische Motive. Die Entwicklung eines Nuklearwaffenarsenals wird als materielle Manifestation der Juche-Ideologie stilisiert, die seit Jahrzehnten den ideologischen Unterbau des Kim-Regimes bildet. Im Kern dieser Ideologie steht das Streben nach vollkommener Eigenständigkeit in ökonomischen, politischen und militärischen Belangen sowie vor allem eine Konzentration auf die Streitkräfte als Machtbasis des Kim-Regimes (French 2005, S. 30-46). Der Ausbau eines Nuklearwaffenarsenals soll demonstrieren, dass Nordkorea gemäß den Prämissen der Juche-Ideologie aus eigener Kraft und gegen erheblichen Widerstand von außen technologische Höchstleistungen erreichen und die Unabhängigkeit des Staates gewährleisten kann. Das Geistesprodukt von Kim Il-sung und Kim Jongil ${ }^{6}$ erscheint dadurch als effektive und wettbewerbsfähige Ideologie bestätigt, die das Land auf Augenhöhe mit den Großmächten zu heben vermag. Gerade ein Regime, dessen Bevölkerung im alltäglichen Leben dramatische Entbehrungen erdulden muss ${ }^{7}$, benötigt dringend solche Mythen des Erfolges.

Zusätzlich zum Erfolg der Staatsideologie soll die Weiterentwicklung von $\mathrm{Nu}-$ klearwaffen auch Kontinuität in der Führung vermitteln. Das Nuklearwaffenprogramm Nordkoreas ist ein Generationenprojekt. Es wurde von Kim Il-sung begonnen und unter Kim Jong-il maßgeblich weiterentwickelt, zu dessen Lebzeiten Nordkorea die ersten beiden Nukleartests in den Jahren 2006 und 2009 durchführte. Unter Kim Jong-un soll das Programm nun seine technologische Reife erfahren. Der jüngste Test dient dem Regime einmal mehr dazu, den in politischen und militärischen Angelegenheiten wenig ausgewiesenen Kim Jong-un als versierten Staatsmann darzustellen, der sein Land in den erlesenen Kreis militärtechnologisch hochentwickelter Staaten zu führen sowie gegen externe Aggressoren zu verteidigen im Stande ist.

\section{Die Strategien, Motive und Möglichkeiten der Großmächte}

Resignation, Gewaltanwendung und Sanktionen als Sackgassen: Strategien im Umgang mit Nordkoreas vehementer Weiterentwicklung des Nuklearwaffen- und Raketenprogramms lassen sich auf einem Spektrum verorten, das von Resignation im Sinn einer Abkehr von Versuchen, das Verhalten Nordkoreas zu beeinflussen, bis zur militärischen Intervention mit dem Ziel eines Regimewechsels reicht. Keiner dieser beiden Pole bietet gegenwärtig eine gangbare Lösung an. Resignation ist vor allem

\footnotetext{
5 Siehe hierzu Manyin und Nikitin (2014).

${ }^{6}$ Wie Paul French (2005, S. 43-44) nachzeichnet, ist die nordkoreanische Propaganda zwar bestrebt, Kim Il-sung und Kim Jong-il als ausschließliche geistige Väter der Juche-Ideologie darzustellen, jedoch scheint ein ehemaliger hochrangiger Funktionär namens Hwang Jang-yop maßgeblichen Einfluss auf deren Entwicklung genommen zu haben.

${ }^{7}$ So sagte etwa der ehemalige Generalsekretär der Vereinten Nationen, Ban Ki-moon, im Februar 2016 8 Mio. US-\$ aus dem UN Central Emergency Response Funds als Soforthilfe für unterernährte Frauen und Kinder in Nordkorea zu.
} 
für die beteiligten Großmächte wenig attraktiv, da sie deren Anspruch als Garanten regionaler Sicherheit unterminieren und damit ein zweifelhaftes Signal an weitere, potenzielle Aspiranten militärischer Nukleartechnologie senden würde. Dies würde ebenfalls zu einer neuerlichen Schwächung des nuklearen Nichtverbreitungsregimes beitragen. Des Weiteren gehen die Großmächte davon aus, dass ein Ausbleiben von Antworten auf Nordkoreas Verhalten zu einer neuen Rüstungsdynamik in der Region führen könnte. Sowohl Japan als auch Südkorea haben substanzielle technologische Fähigkeiten und Ressourcen in der zivilen Nutzung der Nuklearenergie und könnten diese in eine militärische Nutzung überführen. In beiden Ländern lassen sich (zumindest vereinzelte) AdvokatInnen für eine solche Nutzung identifizieren (Johnston 2016; Dalton et al. 2016).

Um der Möglichkeit einer nuklearen Rüstungsdynamik in der Region entgegenzuwirken, bemühen sich die Vereinigten Staaten verstärkt um den Ausbau von Raketenabwehrsystemen in Ostasien. Während Japan diesen Bestrebungen offen gegenüber steht, war Südkorea in der Vergangenheit eher verhalten. Angesichts Nordkoreas jüngster Nuklearwaffen- und Raketentests hat Seoul jedoch seine Haltung verändert. Im Juli 2016 einigten sich Südkorea und die USA auf die Stationierung des Terminal High Altitude Defense (THAAD) Systems in der südöstlichen Region Seongju, das Südkoreas bestehende Fähigkeiten im Bereich der Raketenabwehr erheblich erweitern wird ${ }^{8}$. Dagegen regten sich in Südkorea allerdings heftige Proteste. China und Russland verfolgen diese Erweiterung von US-gestützter Raketenabwehr in der Region mit Argwohn, da sie eine (längerfristige) Einschränkung ihrer Zweitschlagfähigkeit befürchten ${ }^{9}$. Beide Staaten protestierten daher heftig gegen die Stationierungsentscheidung. Der Ausbau von Raketenabwehr stellt nicht nur einen weiteren destabilisierenden Faktor in den Großmachtbeziehungen dar, es bleibt letzlich ebenfalls fraglich, ob Raketenabwehr und erweiterte Abschreckung durch die Vereinigten Staaten in der Lage sein werden, die zunehmende Nervosität Japans und Südkoreas angesichts der nordkoreanischen Drohkulisse im Zaum zu halten. Auch Präsident Donald Trumps Wahlkampfrhetorik hinsichtlich eines Rückzugs Amerikas aus der Region und der Möglichkeit einer eigenständigen nuklearen Abschreckungsfähigkeit Japans und Südkoreas hat nicht gerade zur Beruhigung beigetragen.

Eine militärische Intervention gegen Nordkorea ist gleichfalls eine wenig attraktive Strategie, wenngleich Russland jüngst deutlich gemacht hat, dass die Drohung Nordkoreas mit einem präventiven Nuklearschlag eine Rechtsgrundlage für eine militärische Operation gegen das Land darstellen würde (The Guardian 2016a). Zwar hätte das Kim-Regime wohl keinen Bestand gegen eine massive Intervention, jedoch hat es durch seine grenznahe Artillerie und Kommandooperationen die Fähigkeit, Südkorea massiven Schaden zuzufügen. Vor allem aber würde ein militärisch erzwungener Regimewechsel auf heftigen Widerstand Chinas und Russlands stoßen. Die militärische Zerschlagung Nordkoreas würde mit einer Truppenpräsenz der USA

\footnotetext{
8 Südkorea verfügt gegenwärtig über Abwehrsysteme des Typs PAC-2 (Patriot Advanced Capability 2), die auf PAC-3 modernisiert werden sollen und für eine lokal begrenzte Abwehr von Raketen vorgesehen sind. THAAD wird einen größeren Abwehrraum ermöglichen. Japan verfügt gegenwärtig über PAC-3 Systeme und see-gestützte SM-3 Abwehrraketen.

9 Siehe hierzu Panda (2016).
} 
an der Nordgrenze Koreas einhergehen und wäre für China unannehmbar. Russland würde China dabei unterstützen, eine weitere Einkreisung Chinas durch die USA zu verhindern.

Auch eine begrenztere Militäroperation, die - wie im Rahmen des jüngsten Manövers vom März 2016 durch die USA und Südkorea geprobt (Fifield 2016) ausschließlich gegen Nordkoreas Nuklearwaffen- und Raketenprogramm gerichtet wäre, würde wohl militärische Vergeltungsmaßnahmen durch Pjöngjang nach sich ziehen. Gleichzeitig sind die Erfolgsaussichten einer solchen Strategie begrenzt. Es liegt die Vermutung nahe, dass Nordkorea - wie andere Staaten, die sich in der Entwicklung von Nuklearwaffen versuchten - jenseits der bekannten Komponenten des Nuklearprogramms noch weitere, versteckte Anlagen besitzt ${ }^{10}$. Auch die Vereinigten Staaten hegen diese Vermutung, wie aus einem Bericht des State Department vom Juni 2015 hervorgeht, der von einer ,clear likelihood of additional unidentified nuclear facilities" (U.S. Department of State 2015) spricht. Wie im Fall der Angriffe gegen das irakische Nuklearwaffenprogramm während der Operation Desert Storm (Kreps und Fuhrman 2011, S. 177-178), wäre also auch ein Angriff gegen Nordkoreas Nuklearanlagen mit dem Problem der unbekannten Komponenten konfrontiert. Außerdem wäre der Expertise nordkoreanischer WissenschaftlerInnen durch einen militärischen Angriff kaum beizukommen.

Sanktionen gelten als Mittelweg zwischen den beiden Extremen der Resignation und der militärischen Intervention. Russland und China haben sich lange gegen eine scharfe Sanktionierung Nordkoreas gestellt - mit ähnlichen, aber auch unterschiedlichen Motiven. China hatte sich dem Drängen der USA und Japans, scharfe Sanktionen gegen Nordkorea zu beschliessen, zuvor immer widersetzt. Mit dem letzten Nukleartest hat Nordkorea China aber außerordentlich brüskiert, da China die nordkoreanische Führung wiederholt aufgefordert hatte, nukleare oder ballistische Tests zu unterlassen. Nordkorea aber hat mit dem Nukleartest deutlich gemacht, dass es seine Entscheidungen autonom trifft und das, obwohl China für die wirtschaftliche Stabilität in Nordkorea unerlässlich ist - vor allem im Bereich des Energie-, Metall- und Lebensmittelhandels. Nordkoreas Außenhandelsvolumen lag 2014 bei 7,1 Mrd. US-\$; die Importe betrugen 3,99 Mrd. US-\$, die Exporte 3,1 Mrd. US-\$. $2,67 \mathrm{Mrd}$. US-\$ an Exporten wurden mit der Volksrepublik China abgewickelt. Indien lag mit 72 Mio. US-\$ weit abgeschlagen an zweiter Stelle. Auch im Hinblick auf die Importe ist China mit Abstand der wichtigste Handelspartner: 3,49 Mrd. US-\$ an Importen stammten 2014 aus China; Thailand lag mit 107 Mio. US-\$ an zweiter Stelle (The Observatory of Economic Complexity 2016). Die Führung in Peking hielt über den unruhigen Nachbarstaat also nicht nur wirtschaftlich, sondern auch wenn auch immer widerwilliger - politisch ihre schützende Hand.

China ist, wie alle anderen ständigen Mitglieder des Sicherheitsrates der Vereinten Nationen, an einem nicht-nuklearen Korea interessiert. Darauf zielt es nicht zuletzt

\footnotetext{
10 Obwohl externe BeobachterInnen bereits seit den 1990er-Jahren vermutet hatten, dass Nordkorea neben der Gewinnung von Plutonium aus dem Reaktor in Yongbyon auch an der Entwicklung einer Fähigkeit zur Urananreicherung arbeitet, überraschte Pjöngjang im November 2010, indem es dem amerikanischen Wissenschaftler Siegfried Hecker eine neu errichtete Uran-Anreicherungsanlage präsentierte. Siehe hierzu Hecker (2010).
} 
deswegen, weil die Ausweitung der nordkoreanischen nuklearen und ballistischen Fähigkeiten, eine Stärkung der militärischen Präsenz der USA in Ostasien und der Bündnisbeziehungen der USA mit Japan und Südkorea nach sich zieht. Das aber steht den Bemühungen Pekings entgegen, die Handlungsfreiheit im Ost- und Südchinesischen Meer zu erhöhen. Auch fürchtet China, dass der Ausbau der nuklearen Bewaffnung Nordkoreas zu einer atomaren Rüstung in Japan und Südkorea führen könnte. Wie bereits zuvor erwähnt, verfolgt Peking den Ausbau regionaler Raketenabwehr als Reaktion auf das Säbelrasseln Nordkoreas ebenfalls mit zunehmender Sorge.

China sieht sich aber mit einem strategischen Dilemma konfrontiert. Der dokumentierte Wille der nordkoreanischen Führung, die Erwartungen Chinas zu missachten, stärkt die militärische Präsenz der USA in Ostasien. Andererseits aber kann China auch nicht daran interessiert sein, dass das Regime in Nordkorea zusammenbricht. Eine Implosion des Regimes und - damit sehr wahrscheinlich einhergehend - der staatlichen Strukturen in Nordkorea, würde zunächst eine dramatische Flüchtlingsbewegung nach China und nach Südkorea auslösen. Zudem ist keineswegs auszuschließen, dass das nordkoreanische Regime angesichts seines Niederganges eine militärische Konfrontation mit Südkorea beginnen würde. Dazu kommt, dass bei einer Wiedervereinigung der beiden Koreas unter der Führung Südkoreas, die starke militärische Präsenz der USA und des vereinigten Korea an der chinesischen Grenze sehr wahrscheinlich ist. China ist daher an Stabilität und Dialog mit Nordkorea mindestens ebenso interessiert wie an der Denuklearisierung der koreanischen Halbinsel.

Russland hat in letzter Zeit eine vorsichtige Annäherung an Nordkorea eingeleitet. Zum einen, um Einwirkungsmöglichkeiten auf die nordkoreanische Führung zu erhalten, zum anderen, um die Handelsbeziehungen zu beleben. Im Zuge dieser Annäherung wird auch über die Visafreiheit verhandelt. Überdies hat Russland Nordkorea annähernd $90 \%$ der Staatsschulden erlassen und will Raffinerien sowie Heizkraftwerke in Nordkorea modernisieren. Schließlich sollen Investitionen in die Schieneninfrastruktur auf nordkoreanischem Territorium eine Eisenbahn-Transitstrecke zwischen Russland und Südkorea herstellen. Russland hatte also kein Interesse, Nordkorea mit harten Sanktionen zu belegen, plädierte umgekehrt dafür, die SechsParteien-Gespräche wieder aufzunehmen. Daran sind die USA aber nicht interessiert, da sich die Haltung der Administration von Präsident Barack Obama gegenüber Nordkorea bislang auf zwei Grundsätze stützt: Keine Gespräche mit Nordkorea um der Gespräche willen und keine Zugeständnisse an Nordkorea, solange das Land sich unkooperativ zeigt. Ob und inwieweit Präsident Trump eine Änderung der Haltung anstrebt, lässt sich derzeit noch nicht abschätzen.

Als Reaktion auf die vierte nukleare Testexplosion drängten die Vereinigten Staaten, Japan und Südkorea umgehend auf eine neue Runde härterer Sanktionen der Vereinten Nationen. Überraschend haben sich Russland und China bereit erklärt, darüber zu verhandeln; China wohl nicht zuletzt aufgrund der Widersetzlichkeit Nordkoreas, womit vitale chinesische Sicherheitsinteressen Chinas berührt werden. Der UN-Sicherheitsrat hat schließlich am 2. März 2016 die Resolution 2270 beschlossen, die weitreichende Sanktionen, wenn auch Auslegungsmöglichkeiten bei deren Durchsetzung, enthält (Sicherheitsrat der Vereinten Nationen 2016b). Die Mit- 
gliedstaaten der UN werden angehalten, Luft-, See- und Landfracht von und nach Nordkorea zu kontrollieren (Absatz 18). Die Beschaffung von Kohle, Eisen, Eisenerz, Titan, Gold, Vanadium und seltenen Erden soll unterbunden (Absatz 29) und die Lieferung von Luftfahrzeugs- und Raketentreibstoff eingestellt werden (Absatz 31). Zusätzlich zu diesen multilateralen Sanktionen verhängten die USA im Juni 2016 unilaterale Sanktionen gegen Unternehmen und Banken, die mit nordkoreanischen Banken zusammenarbeiten. Diese Unternehmen und Banken verlieren den Zugang zum Finanzmarkt der USA, was in weiterer Folge vor allem chinesische Banken treffen würde. Im Juli 2016 verhängten die USA schließlich weitere Sanktionen gegen Kim Jong-un persönlich.

Wie bei den bisherigen Massnahmen des UN-Sicherheitsrates gegen Nordkorea, bleibt auch diesmal abzuwarten, ob China strikt dafür sorgen wird, dass diese neuen Sanktionen eingehalten werden (Habib 2016). Es ist jedenfalls der Schlüsselfaktor für die Realisierung des Sanktionsregimes, weil nur China dichte ökonomische Beziehungen mit Nordkorea unterhält. Die an harten Sanktionen am meisten interessierten USA haben hingegen kaum Hebel, um Nordkorea ökonomisch unter Druck zu setzen. Im April 2016 verhängte das chinesische Handelsministerium über mehrere nordkoreanische Exportwaren ein bedingtes Handelsembargo. Betroffen davon sind Kohle, Gold, Titan, Eisenerz und seltene Erden, wenn deren Exporterlöse militärisch genutzt würden. China stellt auch die Lieferung von Kerosin für die militärische Luftfahrt in Nordkorea ein. Abzuwarten bleibt schließlich, ob Nordkorea, wie auch in der Vergangenheit, Wege finden wird, die Sanktionen zu umgehen ${ }^{11}$.

Bislang waren die Sanktionen nicht wirksam, in dem Sinne, die nordkoreanische Führung zu einer Verhaltensänderung zu bewegen. Das hängt zum einen mit dem zuvor dargestellten Umstand zusammen, dass die nuklearen militärischen Kapazitäten für das Regime ein derart vitales und existentielles Interesse sind, dass es die Kosten der Sanktionen auf sich nimmt. Zum anderen aber bleiben die Sanktionen noch immer relativ moderat, weil anders die Zustimmung Chinas im Sicherheitsrat nicht zu erreichen gewesen wäre. Mit den Sanktionen kann zwar die Bestrafung Nordkoreas für unerwünschtes Verhalten erreicht werden, aber aus der gelungenen Bestrafung erwächst keine erfolgreiche Denuklearisierung. Im besten Fall konnten bisherige Sanktionen zu einer Verlangsamung des nuklearen Waffenprogrammes beitragen. $\mathrm{Zu}$ diskutieren ist jedenfalls, ob die verhängten Sanktionen noch immer als gezielte Sanktionen gelten können, oder doch als umfassende Sanktionen, die zumindest indirekt die Lebensumstände der gesamten nordkoreanischen Bevölkerung deutlich erschweren. Die nordkoreanische Führung wird ausgabenseitig immer den Streitkräften und dem militärischen Nuklearprogramm Vorrang einräumen. Wenn die Einnahmen Nordkoreas aufgrund der Exportrestriktionen sinken, werden Inves-

\footnotetext{
${ }^{11}$ Der vom UN-Sicherheitsrat mandatierte Expertenbericht zur multilateralen Sanktionierung Nordkoreas hält hierbei folgendes fest: ,[T]here are serious questions about the efficacy of the current United Nations sanctions regime. The Panel's investigations have shown that the Democratic People's Republic of Korea has been effective in evading sanctions and continues to use the international financial system, airlines and container shipping routes to trade in prohibited items. [...] The Panel has consistently highlighted the problems of non-implementation of the resolutions, which allows prohibited activity to continue" (Sicherheitsrat der Vereinten Nationen 2016a, S. 4).
} 
titionen in den zivilen Sektor und damit die Lebensgrundlagen der nordkoreanischen Bevölkerung vorrangig beschnitten.

Die Befürworter harter Sanktionen stehen vor einer riskanten Abwägung. Zum einen ist zu fragen, ob diese Sanktionen nicht derart weitgehend sind, dass der Regimebestand in Nordkorea gefährdet ist. Regimeerosion oder -implosion, so das Argument, könnten angesichts der unabsehbaren Folgewirkungen dieses Prozesses auch nicht im Interesse der USA sein. Befürworter harter Sanktionen meinen dagegen, dass nur diese in der Lage sind, Nordkorea zu einer Verhaltensänderung zu bewegen und die Führung in Pjöngjang zu zwingen, angesichts der Gefährdung der eigenen Existenz Zugeständnisse zu machen. Das käme für die nordkoreanische Führung einer besonderen Dialektik gleich: Die Nuklearwaffen sollen zum Regimeerhalt der nordkoreanischen Führung dienen; durch das Nuklearprogramm werden aber zunehmend harte wirtschaftliche und finanzielle Sanktionen ausgelöst, die die Regimesicherheit erst Recht unterminieren könnten. Niemand kann aber belastbar prognostizieren, wie sich das Kim-Regime in einem derartigen Szenario wirklich verhalten würde.

Engagement als alternative Strategie?: Nachdem Resignation, militärische Eskalation und Sanktionen wenig aussichtsreich sind, stellt sich letztlich die Frage, ob eine Strategie des engagement zu einer maßgeblichen Änderung im Verhalten Nordkoreas führen könnte. Versteht man engagement zunächst im Sinne eines grand bargains, so fällt die Antwort ebenfalls negativ aus. Nordkorea hat in der Vergangenheit wiederholte Male betont, dass nur der Abschluss eines Friedensvertrages (anstelle des Waffenstillstandsabkommens von 1953) die Gegenleistung eines Teststops ermöglichen werde und erst vor dem Hintergrund eines solchen Vertrages über nukleare Abrüstung gesprochen werden könne. Die Vereinigten Staaten bestehen ihrerseits jedoch auf eine umgekehrte Konditionalität: Nur wenn sich Nordkorea zu einer Denuklearisierung bekennt und erkennbare Schritte in diese Richtung unternimmt, kann es Gespräche über einen Friedensvertrag geben. Ein grand bargain ist aber nicht nur aufgrund dieser konträren Konditionalitäten, sondern auch aufgrund des ihm zugrunde liegenden Zieles der unmittelbaren und umfasenden Abrüstung unwahrscheinlich. Nach Jahrzehnten der Wahrnehmung einer existenziellen Bedrohung und einer tiefsitzenden Skepsis (oder Paranoia) gegenüber den Verenigten Staaten wird sich Nordkorea gegenwärtig nicht mit einem Vertrag als alleinige Existenzsicherung zufrieden geben. Wie am Beginn dieses Beitrages dargelegt, sind Nuklearwaffen für das Regime der ultimative Garant souveräner Existenz und damit gegenwärtig nicht verhandelbar. Dies bestätigte auch der Gesandte von Kim Jongun, Ri Su-yong, gegenüber dem chinesischen Präsidenten Xi Jinping im Juni 2016 (Perlez 2016).

Eine alternative Engagement-Strategie müsste demnach von der Prämisse ausgehen, dass (zumindest) auf absehbare Zeit bestenfalls das Ausmaß des nordkoreanischen Arsenals an Nuklearwaffen und Trägerraketen eingehegt werden kann, 
nicht aber dessen Existenz. ${ }^{12}$ Eine solche Engagement-Strategie müsste von einer Übereinkunft ausgehen, in der sich die Parteien auf das Fernziel einer Denuklearisierung der gesamten koreanischen Halbinsel verständigen, um damit eine defacto-Anerkennung Nordkoreas als Nuklearwaffenstaaten zu vermeiden. Für Nordkorea sollte ein solches Bekenntnis keine Schwierigkeit darstellen, hat Kim Jongun auf dem Parteikongress des Mai 2016 doch bekräftigt, dass sein Land sich um globale Abrüstung bemühen werde (The Guardian 2016b). Dieses Ziel einer Denuklearisierung der koreanischen Halbinsel ist zudem in der gemeinsamen Erklärung Nord- und Südkoreas aus dem Jahr 1992 festgeschrieben (Hayes und Hamel-Green 2009, S. 10-11), die nach wie vor rechtskräftigt ist ${ }^{13}$. Ausgehend von dieser Basis müsste eine Engagement-Strategie - ähnlich dem Joint Comprehensive Action Plan, der mit der Islamischen Republik Iran abgeschlossen wurde - mehrere Phasen der Rüstungskontrolle (Dingli 2016; Hecker 2015) mit Schritten positiver Anreize wie Hilfslieferungen, der Aufhebung von Sanktionen und (längerfristig) Gesprächen über einen Friedensvertrag kombinieren.

Eine erste, vertrauensbildende Maßnahme wäre eine Garantie Nordkoreas, auf den Ersteinsatz von Nuklearwaffen zu verzichten, oder diesen zumindest auf den Fall einer Bedrohung der souveränen Existenz einzugrenzen. Auch diese Maßnahme sollte für Nordkorea machbar sein, nachdem die Führung jüngst neben ihrer traditionell eskalierenden Rhetorik auch das Bild eines verantwortungsbewussten Nuklearwaffenstaats vermitteln wollte, der auf den Ersteinsatz von Nuklearwaffen verzichtet ${ }^{14}$. Um die Wahrnehmung einer existenziellen Bedrohung durch die USA einzuhegen, müsste eine no-first-use-policy Nordkoreas jedoch in jedem Fall eine Entsprechung in einer Garantie der Vereinigten Staaten finden, weder Nuklearwaffen gegen Nordkorea einzusetzen noch deren Verwendung anzudrohen. Eine solche Garantie war bereits im Agreed Framework des Jahres 1994 und dem Joint Statement der Sechs-Parteien Gespräche vom September 2005 angelegt (Sigal 2011, S. 34). Darauf aufbauend und ebenfalls auf Nordkoreas Bild des verantwortungsbewussten Nuklearwaffenstaats abzielend, müsste ein zusätzliches Abkommen den Export sensibler Technologien untersagen. Ein umfassender Test-Stop als nächste Phase der Rüstungskontrolle wäre nur möglich, wenn die nordkoreanische Führung einerseits

\footnotetext{
${ }^{12} \mathrm{Zu}$ dieser Einschätzung gelangt auch der damalige National Intelligence Director der USA, James Clapper, im Oktober 2016. Clapper meinte, die Denuklearisierung Nordkoreas sei „probably a lost cause“ und es wäre nur noch eine Begrenzung der nuklearen Rüstungs denkbar (Dyer 2016).

${ }^{13}$ Dieses Ziel ist ebenfalls in der gemeinsamen Erklärung verankert, die im Rahmen der Sechs-Parteien Gespräche im Jahr 2005 gegeben wurde. In dieser heißt es: „The Six Parties unanimously reaffirmed that the goal of the Six-Party Talks is the verifiable denuclearization of the Korean Peninsula in a peaceful manner. The D.P.R.K. committed to abandoning all nuclear weapons and existing nuclear programs and returning, at an early date, to the Treaty on the Nonproliferation of Nuclear Weapons and to IAEA safeguards. The United States affirmed that it has no nuclear weapons on the Korean Peninsula and has no intention to attack or invade the D.P.R.K. with nuclear or conventional weapons. The R.O.K. reaffirmed its commitment not to receive or deploy nuclear weapons in accordance with the 1992 Joint Declaration of the Denuclearization of the Korean Peninsula, while affirming that there exist no nuclear weapons within its territory. The 1992 Joint Declaration of the Denuclearization of the Korean Peninsula should be observed and implemented“ (U.S. Department of State 2005; Hecker 2015).

${ }^{14}$ Dieser Hinweis auf eine No-First-Use-Politik ist sowohl in der Aussendung der nordkoreanischen Nachrichtenagentur zum jüngsten Nuklearwaffentest (Korean Central News Agency 2016a) als auch in der Rede von Kim Jong-un vor dem Parteitag im Mai 2016 zu finden (The Guardian 2016b).
} 
zu der Einschätzung gelangt, dass sie über zuverlässige Nuklearwaffen- und Trägertechnologien verfügt, wofür (zumindest im Bereich der Trägermittel) aber noch weitere Tests notwendig sein werden. Andererseits müsste sich in den vorangehenden Schritten und durch die schrittweise Umsetzung positiver Anreize ein Mindestmaß an Vertrauen zwischen den Parteien herausgebildet haben, damit Nordkorea diesen nächsten Schritt setzen kann. Dieses Vertrauen wäre auch für die nächste Phase von Verhandlungen über eine Obergrenze oder einen cap (Dingli 2016) für Nordkoreas Arsenal an Nuklearwaffen und Trägermittel unabdingbar, der jedenfalls auch Verifikationsmechanismen beinhalten müsste.

In einer weitaus längerfristigeren Perpektive wäre eine langsame Reduktion des nordkoreanischen Nuklearwaffenarsenals eine weitere Phase der Rüstungkontrolle. Diese wäre jedoch mit großer Wahrscheinlichkeit an den Abschluss eines Friedensvertrages und Sicherheitsgarantien und weitere vertrauensbildende Maßnahmen gebunden. Diese Schritte könnten in den Rahmen erweiterter Verhandlungen über eine nuklearwaffenfreie Zone in Nordostasien eingebettet sein, deren Etablierung in der Vergangenheit bereits mehrere Male von regionalen Akteuren - unter anderem vom Kim Il-sung im Jahre 1980 - gefordert wurde (Hayes und Hamel-Green 2009, S. 10-11). Sollte der Engagement-Prozess diesen Punkt erreichen, wäre es auch für die Vereinigten Staaten einfacher, Nordkorea bezüglich eines Friedensvertrages entgegenzukommen ohne als schwach oder erpressbar zu gelten. Nordkorea könnte in einem solchen Prozess seinerseits das nötige Vertrauen entwickeln, das eine schrittweise Abkehr von seiner nuklearen Bewaffnung zumindest als denkmöglich erscheinen lassen kann.

$\mathrm{Ob}$ eine solche Engagement-Strategie überhaupt beginnen und längerfristig Bestand haben kann, hängt jedoch von mindestens drei Faktoren ab. Um multilaterale Gespräche über die Möglichkeit und die Inhalte einer Engagement-Strategie in Gang zu bringen, bedarf es erstens, einer Abkehr der Vereinigten Staaten von ihrer gegenwärtigen Politik der strategic patience, die angesichts der zunehmenden Reife nordkoreanischer Nuklearwaffen und Trägermittel sowie des eskalierenden Verhaltens des Regimes als gescheitert betrachtet werden darf. Anstatt Gespräche mit Nordkorea an Bedigungen zu knüpfen und gleichzeitig zu hoffen, dass sich das Problem durch eine Desintegration des herrschenden Regimes letztlich von selbst löst, sollten sich die Vereinigten Staaten auf Gespräche ohne Vorbedingungen einlassen. Ob Präsident Trump willens und - angesichts des zu erwartenden, heftigen Widerstands im Kongress - fähig sein wird, diese Abkehr zu vollziehen, bleibt abzuwarten. Erschwert wird diese jedenfalls von der Erfahrung, dass sich Nordkorea in der Vergangenheit nach Engagement-Versuchen externer Akteure wiederum wenig kooperativ gezeigt und sich weiter in Richtung einer Nuklearwaffenfähigkeit bewegt hat.

Ein zweiter, wesentlicher Faktor ist, ob eine Engagement-Strategie auch im Fall weiterer nordkoreanischer Tests aufrechterhalten werden könnte. Wie zuvor erwähnt, wird sich die Führung Nordkoreas erst auf Beschränkungen von Tests und Waffenarsenalen einlassen können, wenn sie zu der Einschätzung gelangt, dass ihre Waffentechnologien gesicherte Verteidigung ermöglichen. Dies bedeutet, dass auch während der früheren Phasen einer Engagement-Strategie mit weiteren Tests zumindest im Bereich ballistischer Trägermittel zu rechnen sein wird. Diese würden 
erheblichen Druck auf die Verhandlungen ausüben, da vor allem VerteidigungsFalken eine unmittelbare Bestrafung und einen Abbruch der Engagement-Strategie fordern würden.

Drittens, ist es ebenfalls ungewiss, ob Nordkorea tatsächlich Interesse an einer nachhaltigen Engagement-Strategie hat. Eine wesentliche Daseinsberechtigung des Kim-Regimes ist in einem klaren Feindbild der Vereinigten Staaten und einem permamenten Kriegszustand mit diesem Feind begründet. Es bleibt also die Frage, ob Nordkorea angesichts dieser inneren Logik tatsächlich den Weg in ein normaleres Verhältnis mit den USA bestreiten kann.

\section{Fazit}

Es war das Ziel dieses Beitrages zu untersuchen, ob und wie sich eine weitere Entwicklung des nordkoreanischen Nuklearwaffen- und Raketenprogrammes eindämmen und, bestenfalls, umkehren lässt. Ausgangspunkt der Analyse war eine Auseinandersetzung mit der Motivlage, die die nordkoreanische Führung zur Aneignung von Nuklearwaffen veranlasst. Es zeigte sich hierbei, dass Nuklearwaffen der Abschreckung einer existenziellen Bedrohung durch die Vereinigten Staaten und der Epressung von Zugeständnissen dienen. Gleichzeitig erfüllen diese jedoch auch die Funktion der Herrschaftssicherung, indem sie als Indikator für die Leistungsfähigkeit der Juche-Ideologie und die Kontinuität in der Herrschaft der Kim-Dynastie stehen.

In der Analyse verschiedener Strategien zur Änderung des nordkoreanischen Verhaltens kam der Beitrag zu dem Ergebnis, dass sowohl die gegenwärtige Strategie der Sanktionierung als auch die alternativen Strategien der Resignation und der militärischen Intervention keine gangbaren Wege zu einer Lösung des Problems darstellen. Eine Verschärfung der Sanktionen könnte die Stabilität des Kim-Regimes gefährden, was vor allem gegen die Interessen Chinas läuft, während Resignation jenen Stimmen in Südkorea und Japan in die Hände spielen würde, die eine eigenständige Nuklearwaffenkapazität dieser beiden Länder fordern. Eine militärische Intervention würde schließlich - egal ob umfassend als Regimwechsel oder begrenzt als Operation gegen das Nuklearwaffenprogramm Nordkoreas - ein erhebliches Maß an Eskalation und Zerstörung nach sich ziehen, das nicht im Interesse der Großmächte ist.

Als Alternative zu diesen Strategien skizziert der Beitrag eine stufenweise Engagement-Strategie. Diese müsste von der Prämisse ausgehen, dass gegenwärtig keine unmittelbare und umfassende Abrüstung Nordkoreas möglicht ist. Eine EngagementStrategie wäre demnach nicht an der Existenz des nordkoreanischen Nuklearwaffenarsenals ausgerichtet, sondern würde zunächst versuchen dessen Ausmaß schrittweise, durch eine Kombination positiver Anreize und sich intensivierender Rüstungskontroll-Maßnahmen, einzuhegen. Als längerfristiges Ziel müsste gleichwohl eine Reduktion des Arsenals und letztlich die Denuklearisierung der koreanischen Halbinsel bestehen bleiben. Auch diese Strategie ist mit Unwegsamkeiten konfrontiert, jedoch bietet sie zum gegenwärtigen Zeitpunkt wohl die einzige Möglichkeit, 


\section{Nordkoreas Verhalten im Sinne regionaler Stabilität und globaler Nichtverbreitung in den Griff zu bekommen.}

Open access funding provided by University of Innsbruck and Medical University of Innsbruck.

Open Access Dieser Artikel wird unter der Creative Commons Namensnennung 4.0 International Lizenz (http://creativecommons.org/licenses/by/4.0/deed.de) veröffentlicht, welche die Nutzung, Vervielfältigung, Bearbeitung, Verbreitung und Wiedergabe in jeglichem Medium und Format erlaubt, sofern Sie den/die ursprünglichen Autor(en) und die Quelle ordnungsgemäß nennen, einen Link zur Creative Commons Lizenz beifügen und angeben, ob Änderungen vorgenommen wurden.

\section{Literatur}

Bleiker, R. (2003). A rogue is a rogue is a rogue: US foreign policy and the Korean nuclear crisis. International Affairs, 79(4), 719-737.

Chanlett-Avery, E., Rinehart, I.E., \& Nikitin, M.B.D. (2016). North Korea: U.S. relations, nuclear diplomacy, and internal situation. Congressional Research Service. https://www.fas.org/sgp/crs/nuke/ R41259.pdf. Zugegriffen: 22. Nov. 2016.

Cordesman, A.H., \& Lin, A. (2015). The changing military balance in the Koreas and North East Asia. Lanham: Rowman \& Littlefield.

Dalton, T., Sunggee, B., \& Tae, L.S. (2016). South Korea debates nuclear options. Carnegie Endowment for International Peace. http://ceip.org/1rgfUrn. Zugegriffen: 3. Nov. 2016.

Dingli, S. (2016). Pragmatism, principle, and the North Korean dilemma. Bulletin of the Atomic Scientists. http://thebulletin.org/north-koreas-nuclear-weapons-what-now/pragmatism-principle-and-northkorean-dilemma. Zugegriffen: 3. Nov. 2016.

Dyer, G. (2016). US spy chief admits North Korea policy has failed. The Financial Times. http://on.ft.com/ 2fUmbYQ. Zugegriffen: 3. Nov. 2016.

Fifield, A. (2016). In drills, U.S., South Korea practice striking North Korea's nuclear plants, leaders. The Washington Post. http://wapo.st/1OXb0nE. Zugegriffen: 3. Nov. 2016.

French, P. (2005). North Korea: The paranoid peninsula - A modern history. London: Zed Books.

Gusterson, H. (1999). Nuclear weapons and the other in Western imagination. Cultural Anthropology, 14(1), 111-143.

Habib, B. (2016). The enforcement problem in Resolution 2094 and the United Nations Security Council sanctions regime: Sanctioning North Korea. Australian Journal of International Affairs, 70(1), 50-68.

Hayes, P., \& Hamel-Green, M. (2009). The path not taken, the way still open: Denuclearizing the Korean peninsula and northeast Asia. The Asia-Pacific Journal, 50(1), 1-24.

Hecker, S.S. (2010). A return trip to North Korea's Yongbyon nuclear complex. Center for International Security and Cooperation, Stanford University. http://cisac.fsi.stanford.edu/sites/default/files/ HeckerYongbyon.pdf. Zugegriffen: 3. Nov. 2016.

Hecker, S.S. (2015). The real threat from North Korea is the nuclear arsenal built over the last decade. Bulletin of the Atomic Scientists. http://thebulletin.org/real-threat-north-korea-nuclear-arsenalbuilt-over-last-decade7883. Zugegriffen: 3. Nov. 2016.

Hymans, J.E.C. (2006). The psychology of nuclear proliferation: Identity, emotions and foreign policy. Cambridge: Cambridge University Press.

IISS - International Institute for Strategic Studies. (2016). The military balance 2016. London: Routlegde.

Johnston, E. (2016). Statements by lawmakers cloud Japan's position on nuclear arms. The Japan Times. http://bit.ly/1tglfjN. Zugegriffen: 3. Nov. 2016.

Korean Central News Agency. (2016a). DPRK proves successful in H-bomb test. http://www.kcnawatch. co/newstream/1452124925-460994118/dprk-proves-successful-in-h-bomb-test/. Zugegriffen: 3. Nov. 2016.

Korean Central News Agency. (2016b). The DPRK succeeded in its first H-bomb test. http://www. kcnawatch.co/newstream/1452247290-841802375/kcna-commentary-lauds-successful-h-bombtest-in-dprk/ (Erstellt: Jan.). Zugegriffen: 3. Nov. 2016.

Kreps, S. E., \& Fuhrman, M. (2011). Attacking the atom: Does bombing nuclear facilities affect proliferation? Journal of Strategic Studies, 34(2), 161-187.

Manyin, M.E., \& Nikitin, M.B.D. (2014). Foreign assistance to North Korea. Congressional Research Service. http://www.fas.org/sgp/crs/row/R40095.pdf. Zugegriffen: 22. Nov. 2016. 
Panda, A. (2016). What is THAAD, what does it do, and why is China mad about it? The Diplomat. http:// bit.ly/1SYuC2i. Zugegriffen: 24. Juni 2016.

Paul, T. V. (2000). Power versus prudence: Why nations forgo nuclear weapons. Montreal: McGill-Queen's University Press.

Perlez, J. (2016). North Korea tells China of 'permanent' nuclear policy. The New York Times. https:// www.nytimes.com/2016/06/01/world/asia/china-north-korea-ri-su-yong.html?_r=0. Zugegriffen: 11. Jan. 2017.

Pyongyang Times. (2016). DPRK's H-bomb test logical chain of reaction. http://www.naenara.com.kp/en/ order/pytimes/?page=World\&no=21409. Zugegriffen: 3. Nov. 2016.

Sagan, S. D. (1996). Why do states build nuclear weapons?: Three models in search for a bomb. International Security, 21(3), 54-88.

Sang-Hun, C. (2016). North Korean propaganda video depicts nuclear strikes on Washington. The New York Times. http://nyti.ms/1pCSJHk. Zugegriffen: 3. Nov. 2016.

Senn, M. (2013). Linguistische Ansätze und die demand-side nuklearer Proliferation: Eine programmatische Bestandsaufnahme. Österreichische Zeitschrift für Politikwissenschaft, 42(2), 197-211.

Sicherheitsrat der Vereinten Nationen. (2016a). Report of the panel of experts established pursuant to resolution 1874. http://bit.ly/1Ogfaxx. Zugegriffen: 24. Juni 2016.

Sicherheitsrat der Vereinten Nationen. (2016b). Security Council imposes fresh sanctions on Democratic People's Republic of Korea, unanimously adopting Resolution 2270. http://www.un.org/press/en/ 2016/sc12267.doc.htm. Zugegriffen: 3. Nov. 2016.

Sigal, L. V. (2011). Political prospects for a nuclear-weapons-free zone in northeast Asia. Pacific Focus, 26(1), 22-36.

Solingen, E. (2007). Nuclear logics: Contrasting paths in east Asia and the Middle East. Princeton: Princeton University Press.

Stimme Koreas. (2016). North Korean video shows nuclear destruction of Washington D.C. https://www. youtube.com/watch?v=Alszb3bLhPw. Zugegriffen: 22. Nov. 2016.

The Guardian. (2016a). Russia warns North Korea over threats of a nuclear strike. http://www.theguardian. com/world/2016/mar/08/russia-warns-north-korea-nuclear-strike. Zugegriffen: 3. Nov. 2016.

The Guardian. (2016b). North Korea will not use nuclear weapons first, says Kim Jong-un. http://bit.ly/ 28RY76k. Zugegriffen: 3. Nov. 2016.

The Observatory of Economic Complexity. (2016). North Korea. http://atlas.media.mit.edu/en/profile/ country/prk/. Zugegriffen: 3. Nov. 2016.

U.S. Department of State. (2005). Joint statement of the fourth round of the six-party talks, Beijing, 19 September 2005. http://www.state.gov/p/eap/regional/c15455.htm. Zugegriffen: 3. Nov. 2016.

U.S. Department of State. (2015). 2015 Report on adherence to and compliance with arms control, nonproliferation, and disarmament agreements and commitments. http://www.state.gov/t/avc/rls/rpt/2015/ 243224.htm. Zugegriffen: 22. Nov. 2016.

Waltz, K. N. (1981). The spread of nuclear weapons: More may be better (Adelphi 171). London: International Institute for Strategic Studies. 